\title{
ON ODD PERFECT NUMBERS
}

\author{
FENG-JUAN CHEN and YONG-GAO CHEN ${ }^{凶}$
}

\author{
(Received 14 December 2011)
}

\begin{abstract}
Let $q$ be an odd prime. In this paper, we prove that if $N$ is an odd perfect number with $q^{\alpha} \| N$ then $\sigma\left(N / q^{\alpha}\right) / q^{\alpha} \neq p, p^{2}, p^{3}, p^{4}, p_{1} p_{2}, p_{1}^{2} p_{2}$, where $p, p_{1}, p_{2}$ are primes and $p_{1} \neq p_{2}$. This improves a result of Dris and Luca ['A note on odd perfect numbers', arXiv:1103.1437v3 [math.NT]]: $\sigma\left(N / q^{\alpha}\right) / q^{\alpha} \neq$ $1,2,3,4,5$. Furthermore, we prove that for $K \geq 1$, if $N$ is an odd perfect number with $q^{\alpha} \| N$ and $\sigma\left(N / q^{\alpha}\right) / q^{\alpha} \leq K$, then $N \leq 4^{K^{8}}$.

2010 Mathematics subject classification: primary 11A05; secondary 11A25.

Keywords and phrases: odd perfect number, primitive prime factor.
\end{abstract}

\section{Introduction}

For a positive integer $N$, let $\sigma(N)$ be the sum of all positive divisors of $N$. We call $N$ perfect if $\sigma(N)=2 N$. It is well known that an even integer $N$ is perfect if and only if $N=2^{p-1}\left(2^{p}-1\right)$, where $p$ and $2^{p}-1$ are both primes. It is not known whether or not odd perfect numbers exist. If such a number $N$ exists, it must have the form $N=p^{\alpha} q_{1}^{2 \beta_{1}} \cdots q_{t}^{2 \beta_{t}}$, where $p, q_{1}, \ldots, q_{t}$ are primes and $p \equiv \alpha \equiv 1(\bmod 4)$. This was proved by Euler in 1849. Recently, Ochem and Rao [6] showed that there is no odd perfect number below $10^{1500}$. Moreover, it has been proved that an odd perfect number must have at least nine distinct prime factors (see [5]).

Suppose that $N$ is a perfect number with $q^{\alpha} \| N$, where $q$ is prime and $q^{\alpha} \| N$ means that $q^{\alpha} \mid N$ and $q^{\alpha+1} \nmid N$. Since $\sigma(N)=2 N$,

$$
\sigma\left(\frac{N}{q^{\alpha}}\right) \sigma\left(q^{\alpha}\right)=\sigma(N)=2 N=2 q^{\alpha} \frac{N}{q^{\alpha}} .
$$

Since $\left(q^{\alpha}, \sigma\left(q^{\alpha}\right)\right)=1$,

$$
q^{\alpha} \mid \sigma\left(\frac{N}{q^{\alpha}}\right)
$$

This work was supported by the National Natural Science Foundation of China (Grant No. 11071121) and the Project of Graduate Education Innovation of Jiangsu Province (CXZZ11-0868).

(C) 2012 Australian Mathematical Publishing Association Inc. 0004-9727/2012 \$16.00 
and $\sigma\left(N / q^{\alpha}\right) / q^{\alpha}$ is a divisor of $2 N$. If $N$ is an even perfect number with $q^{\alpha} \| N$, then $\sigma\left(N / q^{\alpha}\right) / q^{\alpha}=1$ or 2 . If $N$ is an odd perfect number and $q^{\alpha} \| N$, then by (1.1), $4 \nmid \sigma\left(N / q^{\alpha}\right) / q^{\alpha}$.

In the following, we always assume that $q$ is an odd prime. Recently, Dris and Luca [3] posed a new approach to research on odd perfect numbers and proved the following results.

Theorem A. If $N$ is an odd perfect number with $q^{\alpha} \| N$, then $\sigma\left(N / q^{\alpha}\right) / q^{\alpha} \notin$ $\{1,2,3,4,5\}$.

THEOREM B. For every fixed $K>5$, there are only finitely many odd perfect numbers $N$ such that, for some prime power $q^{\alpha} \| N, \sigma\left(N / q^{\alpha}\right) / q^{\alpha}<K$. All such $N$ are bounded by some effectively computable number depending on $K$.

For a positive integer $n$ with the standard factorisation $n=p_{1}^{\alpha_{1}} \cdots p_{s}^{\alpha_{s}}\left(\alpha_{i}>0, i=\right.$ $1,2, \ldots, s)$, let $\Omega(n)=\alpha_{1}+\cdots+\alpha_{s}$ and $\omega(n)=s$.

In this paper, we improve the above results by proving the following theorems.

Theorem 1.1. Suppose that $N$ is an odd perfect number with $q^{\alpha} \| N$. Let $m=$ $\sigma\left(N / q^{\alpha}\right) / q^{\alpha}$. Then

$$
\Omega(m)+\omega(m) \geq \omega(N)-\log _{2} \omega(N),
$$

where $\log _{2}$ means the logarithm to base 2 .

From $\omega(N) \geq 9$ and Theorem 1.1, we immediately have the following corollary.

Corollary 1.2. Suppose that $N$ is an odd perfect number with $q^{\alpha} \| N$. Let $m=$ $\sigma\left(N / q^{\alpha}\right) / q^{\alpha}$. Then $\Omega(m)+\omega(m) \geq 6$. That is,

$$
m \neq p, p^{2}, p^{3}, p^{4}, p_{1} p_{2}, p_{1}^{2} p_{2},
$$

where $p, p_{1}, p_{2}$ are primes and $p_{1} \neq p_{2}$.

Theorem 1.3. Suppose that $K \geq 1$ and $N$ is an odd perfect number. If $q^{\alpha} \| N$ with $\sigma\left(N / q^{\alpha}\right) / q^{\alpha} \leq K$, then $N \leq 4^{K^{8}}$.

REMARK 1.4. From a detailed proof of Theorem 1.3, we can in fact show that $N \leq 4^{K^{\theta}}$, where $\theta=\log 4 / \log 3+o(1)$.

\section{Preliminary lemmas}

Suppose that $N$ is an odd perfect number, so $\sigma(N)=2 N$. Write

$$
N=p_{1}^{\lambda_{1}} p_{2}^{\lambda_{2}} \cdots p_{s}^{\lambda_{s}} q^{\alpha},
$$

where the primes $p_{1}, p_{2}, \ldots, p_{s}, q$ are distinct odd numbers and not necessarily ordered increasingly. Let

$$
\sigma\left(p_{i}^{\lambda_{i}}\right)= \begin{cases}m_{i} q^{\beta_{i}} & i=1,2, \ldots, k, \\ q^{\beta_{i}} & i=k+1, \ldots, s,\end{cases}
$$


where $m_{i} \geq 2$, and $q \nmid m_{i}$ for $i=1,2, \ldots, k$. We put $m=m_{1} m_{2} \cdots m_{k}$ and $t=\omega_{0}(m)$; $\omega_{0}(m)$ is the number of distinct odd prime factors of $m$. It is clear that $k \leq \Omega(m)$.

Since $\sigma(N)=2 N$,

$$
\sigma\left(p_{1}^{\lambda_{1}}\right) \cdots \sigma\left(p_{s}^{\lambda_{s}}\right) \sigma\left(q^{\alpha}\right)=2 N=2 p_{1}^{\lambda_{1}} p_{2}^{\lambda_{2}} \cdots p_{s}^{\lambda_{s}} q^{\alpha} .
$$

That is,

$$
m q^{\beta_{1}+\beta_{2}+\cdots+\beta_{s}} \sigma\left(q^{\alpha}\right)=2 p_{1}^{\lambda_{1}} p_{2}^{\lambda_{2}} \cdots p_{s}^{\lambda_{s}} q^{\alpha} .
$$

By $q \nmid \sigma\left(q^{\alpha}\right)$ and $q \nmid m$, we have $\alpha=\beta_{1}+\beta_{2}+\cdots+\beta_{s}$. Hence,

$$
m \sigma\left(q^{\alpha}\right)=m \frac{q^{\alpha+1}-1}{q-1}=2 p_{1}^{\lambda_{1}} \cdots p_{k}^{\lambda_{k}} p_{k+1}^{\lambda_{k+1}} \cdots p_{s}^{\lambda_{s}}=\frac{2 N}{q^{\alpha}} .
$$

By (1.1) and (2.2),

$$
m=\frac{2 N}{q^{\alpha} \sigma\left(q^{\alpha}\right)}=\frac{\sigma\left(N / q^{\alpha}\right)}{q^{\alpha}} .
$$

Definition 2.1. A prime factor $p$ of $a^{n}-1$ is called primitive if $p \nmid a^{j}-1$ for all $0<j<n$.

Our proofs are based on the following lemmas.

Lemma $2.2[1,2,7]$. Let $a$ and $n$ be integers greater than 1 . There exists a primitive prime factor of $a^{n}-1$, except precisely in the following cases: (i) $n=2, a=2^{\beta}-1$, where $\beta \geq 2$; (ii) $n=6, a=2$.

Lemma 2.3 [3]. Let $\lambda, \alpha, \beta$ be positive integers, and $p, q$ be primes such that

$$
\frac{p^{\lambda+1}-1}{p-1}=q^{\beta}, \quad p^{\lambda} \mid \frac{q^{\alpha+1}-1}{q-1} .
$$

Then $p^{\lambda-1} \mid \alpha+1$.

Let $d(\alpha+1)$ denote the number of positive divisors of $\alpha+1$.

Lemma 2.4. Let $N$ be an odd perfect number with $q^{\alpha} \| N$. Then $d(\alpha+1) \leq \omega(N)$.

Proof. Let $n_{1}, n_{2}, \ldots, n_{w}$ be all of the distinct divisors of $\alpha+1$ which are larger than 1 . If $2 \mid \alpha+1$, then by Euler's result we have $q \equiv \alpha \equiv 1(\bmod 4)$. Thus, by Lemma 2.2, for each $1 \leq i \leq w$, there exists a primitive prime factor $q_{i}$ of $q^{n_{i}}-1$. Since $2 \mid q-1$ and $n_{1}, n_{2}, \ldots, n_{w}$ are distinct and larger than 1 , we know that $q_{1}, \ldots, q_{w}$ are distinct odd primes. Noting that $n_{1}, n_{2}, \ldots, n_{w}$ are divisors of $\alpha+1$,

$$
q^{n_{i}}-1 \mid q^{\alpha+1}-1, \quad 1 \leq i \leq w .
$$

Hence,

$$
q_{1} \cdots q_{w} \mid \frac{q^{\alpha+1}-1}{q-1} .
$$

By (2.2), we have $d(\alpha+1)=w+1 \leq s+1=\omega(N)$. 


\section{Proofs of theorems}

Proof of Theorem 1.1. If $\left(m, p_{k+1} \cdots p_{s}\right)=p_{k+1} \cdots p_{s}$, then $s-k \leq t$. So

$$
k+t \geq s=\omega(N)-1 \text {. }
$$

Since $k+t \leq \Omega(m)+\omega(m)$ and $\omega(N) \geq 2$,

$$
\Omega(m)+\omega(m) \geq \omega(N)-1 \geq \omega(N)-\log _{2} \omega(N) .
$$

If $\left(m, p_{k+1} \cdots p_{s}\right) \neq p_{k+1} \cdots p_{s}$, without loss of generality, we may assume that

$$
\frac{p_{k+1} \cdots p_{s}}{\left(m, p_{k+1} \cdots p_{s}\right)}=p_{l+1} \cdots p_{s}, \quad k \leq l<s .
$$

By (2.2) and (3.1),

$$
p_{l+1}^{\lambda_{l+1}} \cdots p_{s}^{\lambda_{s}} \mid \sigma\left(q^{\alpha}\right)
$$

Using (2.1) and Lemma 2.3,

$$
p_{i}^{\lambda_{i}-1} \mid \alpha+1, \quad i=l+1, \ldots, s .
$$

So

$$
p_{l+1}^{\lambda_{l+1}-1} \cdots p_{s}^{\lambda_{s}-1} \mid \alpha+1 .
$$

Since $\sigma\left(p_{i}^{\lambda_{i}}\right)=q^{\beta_{i}}$ and $2 \nmid q$, we have that $\lambda_{i}$ is even for $k+1 \leq i \leq s$. Thus, $\lambda_{i} \geq 2$ for $l+1 \leq i \leq s$ and then $p_{l+1} \cdots p_{s} \mid \alpha+1$.

Case 1. $2 \nmid m$. By (2.2) and

$$
\frac{q^{\alpha+1}-1}{q-1}=q^{\alpha}+\cdots+q+1 \equiv \alpha+1 \quad(\bmod 2),
$$

we have $2 \mid \alpha+1$. Thus, $2 p_{l+1} \cdots p_{s} \mid \alpha+1$. By Lemma 2.4,

$$
2^{s-l+1} \leq d(\alpha+1) \leq \omega(N) .
$$

That is,

$$
s-l+1 \leq \log _{2} \omega(N)
$$

Thus

$$
l \geq \omega(N)-\log _{2} \omega(N) .
$$

By $\omega_{0}(m)=t$ and (3.1), we have $l-k \leq t$. So $l \leq k+t \leq \Omega(m)+\omega(m)$. Hence,

$$
\Omega(m)+\omega(m) \geq \omega(N)-\log _{2} \omega(N) .
$$

Case 2. $2 \mid m$. Since $p_{l+1} \cdots p_{s} \mid \alpha+1$,

$$
2^{s-l} \leq d(\alpha+1) \leq \omega(N)
$$

So

$$
l \geq s-\log _{2} \omega(N)=\omega(N)-\log _{2} \omega(N)-1 .
$$


In a similar manner to case 1 ,

$$
l \leq k+t \leq \Omega(m)+\omega_{0}(m)=\Omega(m)+\omega(m)-1 .
$$

Hence

$$
\Omega(m)+\omega(m) \geq \omega(N)-\log _{2} \omega(N) .
$$

This completes the proof of Theorem 1.1.

Proof of Theorem 1.3. Since $m=m_{1} m_{2} \cdots m_{k}=\sigma\left(N / q^{\alpha}\right) / q^{\alpha} \leq K$, we have $\omega(m) \leq$ $\Omega(m) \leq \log K / \log 2$. By Theorem 1.1,

$$
\frac{2 \log K}{\log 2} \geq \Omega(m)+\omega(m) \geq \omega(N)-\log _{2} \omega(N) .
$$

Since $\omega(N) \geq 9$,

$$
\log _{2} \omega(N) \leq \frac{1}{2} \omega(N)
$$

Thus, $\omega(N) \leq 4 \log K / \log 2$. Using a famous result of Heath-Brown [4],

$$
N<4^{4^{\omega(N)}} \leq 4^{4^{4 \log K / \log 2}}=4^{K^{8}} .
$$

This completes the proof of Theorem 1.3.

\section{Acknowledgement}

We are grateful to the referee for his/her helpful comments.

\section{References}

[1] A. S. Bang, 'Taltheoretiske undersøgelser', Tidsskrift for Mat. 5(4) (1886), 70-80, 130-137.

[2] G. D. Birkhoff and H. S. Vandiver, 'On the integral divisors of $a^{n}-b^{n}$, Ann. of Math. (2) 5 (1904), 173-180.

[3] J. A. B. Dris and F. Luca, 'A note on odd perfect numbers', arXiv:1103.1437v3 [math.NT].

[4] D. R. Heath-Brown, 'Odd perfect numbers', Math. Proc. Cambridge Philos. Soc. 115 (1994), 191-196.

[5] P. P. Nielsen, 'Odd perfect numbers have at least nine prime factors', Math. Comp. 76 (2007), 2109-2126.

[6] P. Ochem and M. Rao, 'Odd perfect numbers are greater than $10^{1500}$, Math. Comp., in press.

[7] M. Roitman, 'On Zsigmondy primes', Proc. Amer. Math. Soc. 125 (1997), 1913-1919.

FENG-JUAN CHEN, School of Mathematical Sciences and Institute of Mathematics, Nanjing Normal University, Nanjing 210046, PR China

and

Department of Mathematics, Suzhou University,

Suzhou 215006, PR China

YONG-GAO CHEN, School of Mathematical Sciences and Institute of Mathematics, Nanjing Normal University, Nanjing 210046, PR China

e-mail: ygchen@njnu.edu.cn 